\title{
Correspondence regarding “Delamanid for rifampicin-resistant tuberculosis: a retrospective study from South Africa"
}

\author{
To the Editor:
}

Here we report on the final outcomes for the cohort of 103 patients, 77\% HIV-positive with a median (interquartile range, IQR) CD4 count of 141 (61-252) cells per $\mathrm{mm}^{3}$, from Khayelitsha, South Africa who initiated regimens containing delamanid $(\mathrm{Dlm})$ for the treatment of rifampicin-resistant tuberculosis (RR-TB) from November 2015 to August 2017 [1]. The median (IQR) duration on Dlm was 6.3 (4.312.0) months; 54 (52\%) patients received Dlm for more than 6 months and $32(31 \%)$ also received a regimen containing bedaquiline (Bdq), $26(81 \%)$ of whom harboured fluoroquinolone (Fq) resistant strains.

Overall, 57 (55\%), $22(21 \%), 14(14 \%)$, six (6\%), and four (4\%) patients had treatment success (cured/ completed treatment), loss to follow-up, death, failure of treatment, and transfer-out, respectively. The median (IQR) times to poor outcomes were as follows: loss to follow-up 10.4 (4.6-16.8) months, death 4.6 (3.3-14.3) months, and the failure of treatment $14.2(9.2-16.7)$ months. Three (21\%) and $10(71 \%)$ of the deaths occurred within 3 and 6 months of treatment initiation $(p=0.021)$. Four of the six cases with failure of treatment (all of whom were HIV-positive) had received Dlm due either to previous RR-TB treatment failure or limited therapeutic options; additionally, four cases received Dlm for $>6$ months due to delayed treatment response. Two of these patients had culture converted to negative and thereafter reconverted to positive while the remaining four never culture converted to negative. At the time in which the outcome of failure was assigned, four of the six cases were no longer on Dlm. There were no significant differences in final outcomes among patients with Fq-resistant compared to Fq-susceptible strains (66\% versus 51\%; $\mathrm{p}=0.33)$.

As we were unable to confirm what happened to the four patients transferred out, they were excluded from further analyses, leaving 78 and $21 \mathrm{HIV}$-positive and -negative patients. The rate of unsuccessful outcomes (loss to follow-up, death and failure of treatment) was higher among HIV-positive patients when compared to HIV-negative patients $(\mathrm{n}=38,49 \%$ versus $\mathrm{n}=4,19 \% ; \mathrm{p}=0.024)$. In the unadjusted analysis, male sex and HIV positivity, regardless of CD4 count, aside from those with an unknown CD4 count, were significantly associated with unsuccessful outcomes. After adjustment for potential confounders, male sex and HIV-positivity with a CD4 count $<200$ copies per $\mathrm{mm}^{3}$ remained significantly associated with unsuccessful outcomes (adjusted hazard ratio (aHR) 3.0, 95\% CI 1.4-6.3; p=0.003; and aHR 3.8, 95\% CI $1.2-11.6 ; \mathrm{p}=0.020$; respectively) (table 1). No factors were identified as significantly associated with successful outcomes in the adjusted analysis.

In total, there were 74 serious adverse events (SAEs) experienced by 31 (30\%) patients; the overall incidence was 4.63 SAEs per 100 person months. Of the 74 SAEs, 25 (34\%) were assessed as related to Dlm. The most common SAEs were QTcF prolongation ( $\mathrm{n}=8$ instances), anaemia ( $\mathrm{n}=7$ instances), meningitis ( $n=6$ instances), seizures ( $n=6$ instances), vomiting ( $n=5$ instances), diarrhoea ( $n=3$ instances), psychotic disorder ( $\mathrm{n}=3$ instances), and renal impairment ( $\mathrm{n}=3$ instances). The eight instances of QTcF prolongation reported as serious occurred in eight (8\%) patients, of whom five (63\%) and one (12\%) were receiving the combination of Dlm, Bdq and clofazimine, and Dlm and Bdq, respectively. The frequency of

@ERSpublications

Delamanid (Dlm) containing regimens to treat RR-TB are effective, but more data on Dlm use is needed. Dlm remains an important treatment option in programmatic settings with high rates of HIV co-infection and in persons with limited treatment options. https://bit.ly/3cVLeUq

Cite this article as: Mohr-Holland E, Reuter A, Hughes J, et al. Correspondence regarding "Delamanid for rifampicin-resistant tuberculosis: a retrospective study from South Africa”. Eur Respir J 2020; 56: 2000837 [https://doi.org/10.1183/13993003.00837-2020]. 
TABLE 1 Unadjusted and adjusted analysis of factors associated with time to unsuccessful outcomes among 103 patients from Khayelitsha, South Africa who initiated regimens containing delamanid (Dlm) from November 2015 to August 2017 for the treatment of rifampicin-resistant tuberculosis (RR-TB)

\begin{tabular}{|c|c|c|c|c|c|c|}
\hline & $\begin{array}{l}\text { Unsuccessful } \\
\text { outcome }\end{array}$ & $\begin{array}{l}\text { Successful } \\
\text { outcome }\end{array}$ & $\begin{array}{l}\text { Unadjusted HR } \\
\text { (95\% CI) }\end{array}$ & p-value & $\begin{array}{l}\text { Adjusted HR } \\
\text { (95\% CI) }\end{array}$ & p-value \\
\hline \multicolumn{7}{|l|}{ Sex } \\
\hline Female & $11(26.2)$ & $28(49.1)$ & 1.0 (reference) & & 1.0 (reference) & \\
\hline \multicolumn{7}{|l|}{ Age years } \\
\hline $0-24$ & $2(4.8)$ & $11(19.3)$ & $0.23(0.06-0.97)$ & 0.045 & & \\
\hline \multicolumn{7}{|l|}{ Body mass index $\mathrm{kg} \cdot \mathrm{m}^{-2}$} \\
\hline$<18$ & $10(25.0)$ & $16(25.4)$ & $1.2(0.58-2.4)$ & 0.65 & & \\
\hline$\geqslant 18$ & $26(65.0)$ & $43(68.3)$ & 1.0 (reference) & & & \\
\hline Missing & $4(10.0)$ & $4(6.4)$ & $1.5(0.52-4.3)$ & 0.46 & & \\
\hline \multicolumn{7}{|l|}{ HIV and CD4 cell count per $\mathrm{mm}^{3}$} \\
\hline HIV negative & $4(9.5)$ & $17(29.8)$ & 1.0 (reference) & & 1.0 (reference) & \\
\hline \multicolumn{7}{|l|}{ Resistance classification } \\
\hline RR-TB & $29(69.1)$ & $35(61.4)$ & 1.0 (reference) & & 1.0 (reference) & \\
\hline Fluoroquinolone resistance & $13(31.0)$ & $22(38.6)$ & $0.46(0.22-0.97)$ & 0.042 & $0.44(0.16-1.2)$ & 0.099 \\
\hline \multicolumn{7}{|l|}{ Previous TB treatment } \\
\hline None & 15 (35.7) & $26(45.6)$ & 1.0 (reference) & & & \\
\hline $\begin{array}{l}\text { First- or second-line TB } \\
\text { treatment previously }\end{array}$ & 27 (64.3) & $31(54.4)$ & $1.2(0.65-2.3)$ & 0.54 & & \\
\hline \multicolumn{7}{|c|}{ Received a regimen containing the combination of $\mathrm{Bdq}$ and $\mathrm{DIm}$} \\
\hline Yes & $12(28.6)$ & $19(33.3)$ & 1.0 (reference) & & 1.0 (reference) & \\
\hline No & $30(71.4)$ & $38(66.7)$ & $1.5(0.77-3.1)$ & 0.23 & $0.69(0.28-1.7)$ & 0.42 \\
\hline
\end{tabular}

SAEs reported among HIV-positive and -negative patients did not significantly differ (34\% versus $17 \%$; $\mathrm{p}=0.13)$. Dlm was withdrawn as a result of 13 SAEs in seven (7\%) patients; 41 SAEs were recovered/ resolved while 18 were fatal (two fatal SAEs were assessed as related to Dlm).

Delamanid was classified as a Category C drug in the recent World Health Organization recommendations and constitutes an important drug for those with limited therapeutic options receiving treatment for RR-TB [2]. While the rate of treatment success reported in this cohort is similar to the ones reported globally in RR-TB patients [3], it is relatively low when compared to success rates observed in other cohorts of patients treated with Dlm, mostly in low HIV-prevalence settings $[4,5]$. Our data suggest that HIV infection still plays a leading role in driving unsuccessful outcomes among those treated for RR-TB, despite access to new drugs [3]. The high frequency of patients with advanced HIV disease may explain the high proportion of early deaths at 6 months (71\%). Additionally, the higher frequency of loss to follow-up among HIV-positive patients highlights that the challenges associated with the dual burden of disease, for example pill burden and the occurrence of frequent non-serious side-effects, that might trump treatment completion [6]. Although second-line resistance is known to be associated with poorer outcomes, we did not observe differences in outcomes among patients with more complex resistance profiles, notably $\mathrm{Fq}$ resistance; this is possibly due to the fact that the vast majority (81\%) received regimens containing both Bdq and Dlm. In line with previously reported results [7-9], our data suggest the effective role of the combination in improving outcomes among the most difficult to treat patients.

The RR-TB treatment outcomes among patients receiving Dlm in this cohort were in line with those reported nationally and globally; more programmatic data are needed to better understand the role of Dlm in the treatment of RR-TB [10]. Meanwhile, Dlm remains an important treatment option in programmatic settings with high rates of HIV co-infection and in persons with limited treatment options. Despite improved access to this novel agent, the rates of death and loss to follow-up in this cohort were high, particularly among those HIV co-infected, reflecting the challenges associated with the management of 
comorbidities and the prolonged duration of treatment for RR-TB. Broader and earlier use of new drugs, early diagnosis of advanced HIV disease and early initiation of appropriate antiretroviral therapy, as well as innovative psychosocial interventions, are needed to increase the chance to cure patients with RR-TB and decrease the number of preventable deaths.

Erika Mohr-Holland ${ }^{1,2}$, Anja Reuter ${ }^{1}$, Jennifer Hughes $\odot^{1}$, Johnny Daniels ${ }^{1}$, Busisiwe Beko ${ }^{1}$, Goodman Makhanda ${ }^{3}$, Virginia De Avezedo ${ }^{4}$, Yulene Kock ${ }^{5}$, Helen Cox ${ }^{6}$, Jennifer Furin ${ }^{7}$, Laura Trivino Duran ${ }^{1}$, Petros Isaakidis ${ }^{2}$ and Gabriella Ferlazzo ${ }^{2}$

${ }^{1}$ Médecins Sans Frontières, Operational Centre Brussels (OCB), Khayelitsha Project and Coordination Office, Cape Town, South Africa. ${ }^{2}$ Médecins Sans Frontières, South African Medical Unit (SAMU), Cape Town, South Africa. ${ }^{3}$ South African Medical Research Council, Cape Town, South Africa. ${ }^{4}$ City of Cape Town Health Department, Cape Town, South Africa. ${ }^{5}$ National Department of Health, Pretoria, South Africa. ${ }^{6}$ Institute of Infectious Disease and Molecular Medicine and Wellcome Centre for Infectious Diseases Research in Africa, University of Cape Town, Cape Town, South Africa. ${ }^{7}$ Department of Global Health and Social Medicine, Harvard Medical School, Boston, MA, USA.

Correspondence: Erika Mohr-Holland, Médecins Sans Frontières (MSF) and MSF Southern African Medical Unit, The Isisivana Center, 1 Julius Tsolo Road, Khayelitsha, 7784, South Africa. E-mail: msfocb-khayelitsha-drtb-epi@brussels. msf.org

Received: 24 March 2020 | Accepted after revision: 9 June 2020

Conflict of interest: E. Mohr-Holland has nothing to disclose. A. Reuter has nothing to disclose. J. Hughes has nothing to disclose. J. Daniels has nothing to disclose. B. Beko has nothing to disclose. G. Makanda has nothing to disclose. V. De Azevedo has nothing to disclose. Y. Kock has nothing to disclose. H. Cox reports grants from Wellcome Trust, UK MRC and EDCTP, outside the submitted work. J. Furin has nothing to disclose. L. Trivino Duran has nothing to disclose. P. Isaakidis has nothing to disclose. G. Ferlazzo has nothing to disclose.

\section{References}

1 Mohr E, Hughes J, Reuter A, et al. Delamanid for rifampicin-resistant tuberculosis: a retrospective study from South Africa. Eur Respir J 2018; 51: 1800017.

2 World Health Organization. WHO Consolidated Guidelines on Drug-Resistant Tuberculosis Treatment. Geneva, WHO, 2019.

3 Ahmad N, Ahuja SD, Akkerman OW, et al. Treatment correlates of successful outcomes in pulmonary multidrug-resistant tuberculosis: an individual patient data meta-analysis. Lancet 2018; 392: 821-834.

4 Kuksa L, Barkane L, Hittel N, et al. Final treatment outcomes of multidrug- and extensively drug-resistant tuberculosis patients in Latvia receiving delamanid-containing regimens. Eur Respir J 2017; 50: 2-5.

5 Lee EH, Yong SH, Leem AY, et al. Improved fluoroquinolone-resistant and extensively drug-resistant tuberculosis treatment outcomes. Open Forum Infect Dis 2019; 6: 1-7.

6 Stephens F, Gandhi NR, Brust JCM, et al. Treatment adherence among persons receiving concurrent multidrug-resistant tuberculosis and HIV treatment in KwaZulu-Natal, South Africa. J Acquir Immune Defic Syndr 2019; 82: 124-130.

7 Hafkin J, Hittel N, Martin A, et al. Compassionate use of delamanid in combination with bedaquiline for the treatment of MDR-TB. Eur Respir J 2019; 53: 1801154.

8 Mohr E, Ferlazzo G, Hewison C, et al. Bedaquiline and delamanid in combination for treatment of drug-resistant tuberculosis. Lancet Infect Dis 2019; 19: 470.

9 Olayanju O, Esmail A, Limberis $\mathrm{J}$, et al. A regimen containing bedaquiline and delamanid compared to bedaquiline in patients with drug resistant tuberculosis. Eur Respir J 2020; 55: 1901181.

10 Borisov S, Danila E, Maryandyshev A, et al. Surveillance of adverse events in the treatment of drug-resistant tuberculosis: first global report. Eur Respir J 2019; 54: 1-14. 